\title{
Klein-Gordon equations for toroidal hydromagnetic waves in an axi-symmetric field
}

\author{
J. F. McKenzie ${ }^{1,2}$ and Q. $\mathbf{H u}^{2}$ \\ ${ }^{1}$ Department of Mathematics and Statistics, Durban University of Technology, Steve Biko Campus, Durban, South Africa \\ ${ }^{2}$ CSPAR, University of Alabama, Huntsville, AL, USA
}

Received: 24 April 2009 - Revised: 22 February 2010 - Accepted: 8 March 2010 - Published: 11 March 2010

\begin{abstract}
In this paper we develop the hydromagnetic wave equations for toroidal Alfvén waves in a background axisymmetric magnetic field. In the case where spatial variations are directed along the ambient magnetic field direction, the equations can be cast in a Klein-Gordon form in which the adiabatic-geometric amplitude factor of the perturbations varies as $\sqrt{\rho L^{5}} \sin ^{5} \theta$ along a magnetic field line (where $\theta$ is colatitude and $L$ the $\mathrm{L}$-shell number) and the cutoff frequency, associated with the Klein-Gordon form, displays an astonishing variation with distance along a field line (see Eqs. 35 and 37 of the text), in the case of a dipole magnetic field. We compute the eigenvalues and eigenfunctions for the Earth's dipole field which are relevant to geomagnetic pulsations.
\end{abstract}

Keywords. Magnetospheric physics (MHD waves and instabilities) - Space plasma physics (Experimental and mathematical techniques; Waves and instabilities)

\section{Introduction}

Hydromagnetic theories of geomagnetic pulsations have been developed by Radoski (1967), McClay (1970), and further extended by Southwood (1974) and Chen and Hasegawa (1974). In the latter two papers the background magnetic field geometry was simplified (straight field lines) to bring out more clearly the physics of field line resonance which involves coupling of a fast mode (decaying across the field lines) to a shear Alfvén wave. Tamao (1965) is considered the earliest work in the "conventional field line resonance theory", and he carried out the calculation in a dipole magnetic field. However, it is also interesting to investigate

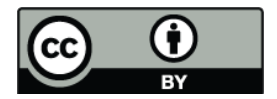

Correspondence to: Q. Hu (qh0001@uah.edu) the effects of plasma inhomogeneity and background magnetic field geometry as developed by Allan and Knox (1979); Walker (1980), and Taylor and Walker (1984). These works provide numerical solutions to the "full wave" equations in a dipole magnetic field geometry.

In this paper, we revisit this problem for the case of toroidal hydromagnetic waves in an axi-symmetric magnetic field (e.g., a dipole). In the next section we derive the azimuthal components of the perturbed (linearized) momentum equation and Faraday's law of induction which provide the wave equations for the perturbed plasma azimuthal velocity $\left(u_{\phi}\right)$ and magnetic field $\left(b_{\phi}\right)$. These equations are equivalent to the wave equations for the electric field given by Cummings et al. (1969) and further developed by Orr and Matthew (1971), and Walker (1980). For spatial variations aligned with the background field the wave equations for $u_{\phi}$ and $b_{\phi}$ can be transformed to the Klein-Gordon (KG) form. This form elucidates two interesting features of the nature of the waveforms. The first feature is that in obtaining the KG form, a certain scaling of the amplitude of the perturbations is effected which implies an adiabatic-geometric growth factor of the perturbation which varies as $\sqrt{\rho L^{5}} \sin ^{5} \theta$ along a magnetic field line (where $\rho$ is the local plasma density, and $L$ is the L-shell number). The variation of the wave amplitude factor with latitude stems from Poynting's theorem which requires the divergence of the wave energy (Poynting) flux through the cross-section of the flux tube to be balanced by the rate of working $\boldsymbol{J} \cdot \boldsymbol{E}$. The other essential feature is the appearance of a cut-off frequency, $\omega_{c}$, characteristic of the KG form, below which waves cannot propagate. The cut-off frequency (slightly different for velocity and magnetic field perturbations) displays a sensitive variation with co-latitude exhibiting a minimum at the equator and attaining a much higher value (about two orders of magnitude) at the ionospheric foot point of the field line. These properties are derived in Sect. 3 and illustrated in Figs. 1 and 2. The eigenvalues (frequencies) and corresponding eigenfunctions

Published by Copernicus Publications on behalf of the European Geosciences Union. 

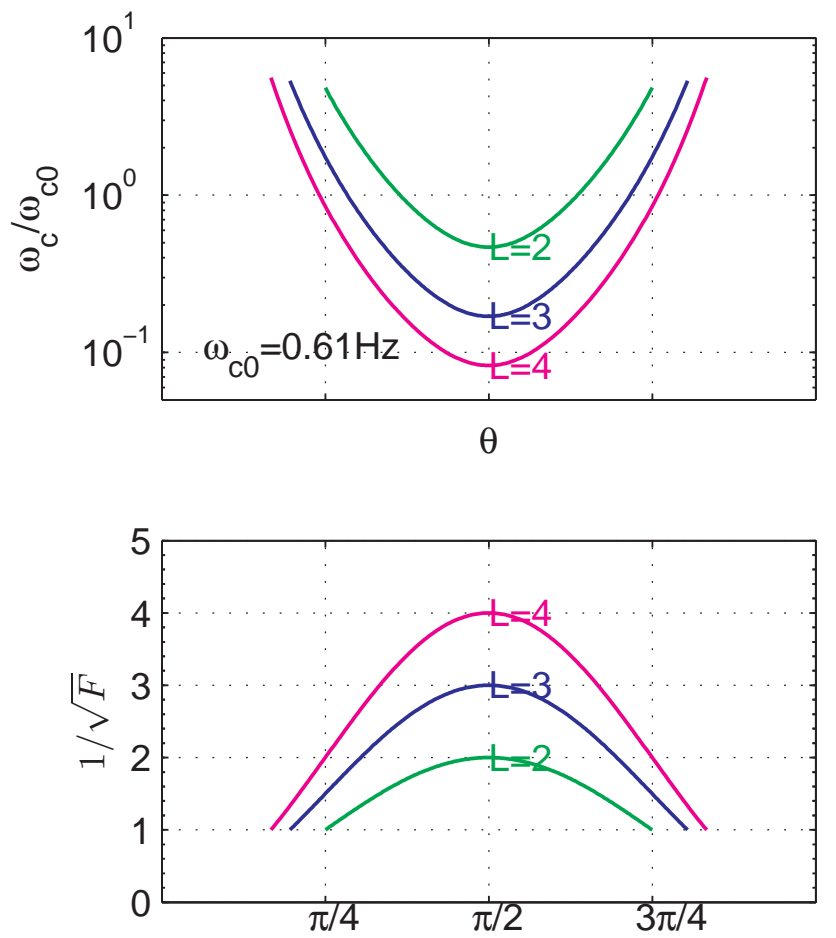

Fig. 1. The parameter $\omega_{c}$ normalized by $\omega_{c 0}$ (with $B_{0}=0.31$ Gauss, $a=6.4 \times 10^{8} \mathrm{~cm}$, and $n_{e}=6.0 \times 10^{4} \mathrm{~cm}^{-3}$ ), and the adiabatic growth/decay factor as a function of $\theta$ for various $L$ values $(p=3)$.

are calculated and shown in Figs. 3 to 7 for the fundamental, first, second, third harmonic and a higher-order harmonic, respectively. We discuss the relevance of these solutions to the phenomenon of geomagnetic pulsations.

\section{The governing equations}

We consider toroidal wave perturbations $\left(b_{\phi}, u_{\phi}\right)$ in the magnetic field and fluid velocity in a background axi-symmetric (that is azimuthal wave number $\mathrm{m}=0$ ) magnetic field $\boldsymbol{B}_{0}=$ $\left(B_{r}, B_{\theta}, 0\right)$. The perturbation electric field $\boldsymbol{E}$ is given by

$\boldsymbol{E}=-\boldsymbol{u} \times \boldsymbol{B}=-u_{\phi} B_{r} \hat{\theta}+u_{\phi} B_{\theta} \hat{r}$

The $\phi$ (toroidal) component of Faraday's law gives

$$
\begin{aligned}
\frac{\partial b_{\phi}}{\partial t} & =\frac{1}{r} \frac{\partial}{\partial r}\left(r B_{r} u_{\phi}\right)+\frac{1}{r} \frac{\partial}{\partial \theta}\left(u_{\phi} B_{\theta}\right) \\
& =\frac{B_{\theta}}{r} \frac{\partial u_{\phi}}{\partial \theta}+B_{r} \frac{\partial u_{\phi}}{\partial r}+\frac{u_{\phi}}{r}\left(\frac{\partial B_{\theta}}{\partial \theta}+\frac{\partial}{\partial r}\left(r B_{r}\right)\right)
\end{aligned}
$$

The equation of a background field line is

$$
\frac{d r}{d \theta}=\frac{r B_{r}}{B_{\theta}}
$$

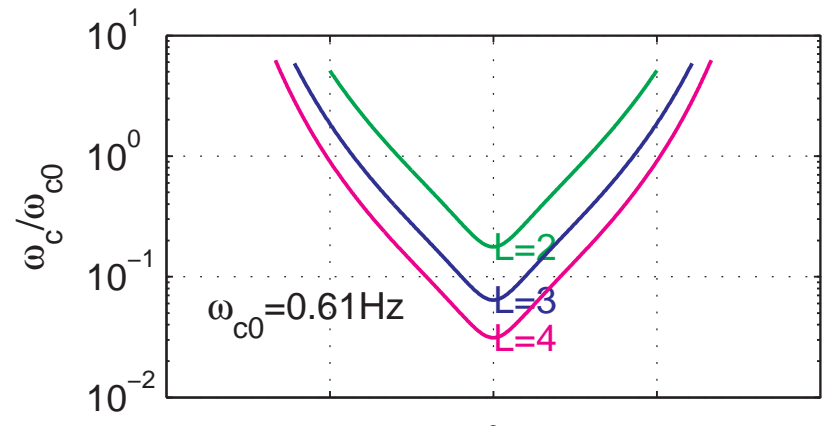

$\theta$

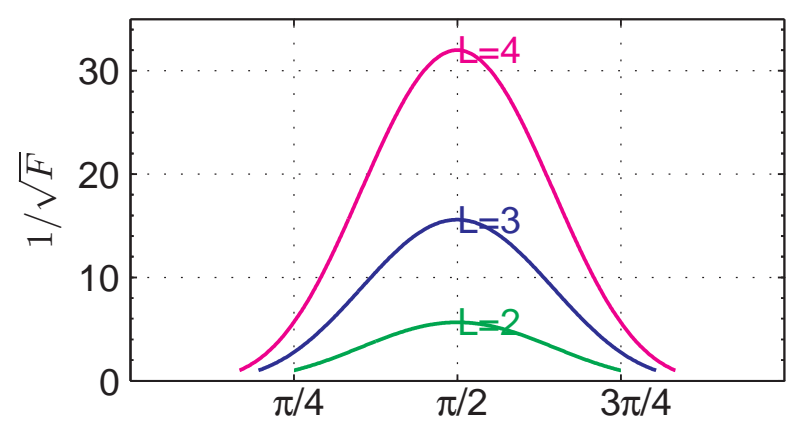

Fig. 2. Same format as Fig. 1 for velocity perturbations.

Therefore, if we restrict our analysis to spatial variations only along field lines as measured by the co-latitude $\theta$, Eq. (2), by using Eq. (3), may be written

$\frac{\partial b_{\phi}}{\partial t}=\frac{2 B_{\theta}}{r} \frac{\partial u_{\phi}}{\partial \theta}+\frac{u_{\phi} B_{\theta}}{r} \frac{1}{l_{b}}$

in which,

$$
\begin{aligned}
\frac{1}{l_{b}} & =\left[\frac{1}{B_{\theta}} \frac{\partial B_{\theta}}{\partial \theta}+\frac{1}{B_{\theta}} \frac{\partial}{\partial r}\left(r B_{r}\right)\right]_{\operatorname{along} B_{0}} \\
& =\frac{d}{d \theta} \ln \left(B_{\theta} g(\theta)\right),
\end{aligned}
$$

where

$\left[\frac{1}{B_{\theta}} \frac{\partial}{\partial r}\left(r B_{r}\right)\right]_{\operatorname{along} B_{0}}=\frac{d}{d \theta} \ln g(\theta)$

The functional form $g(\theta)$ is determined by the model of the background field and will be given subsequently for a dipole.

The $\phi$ (toroidal) component of the momentum equation may similarly be written

$$
\begin{aligned}
\rho \frac{\partial u_{\phi}}{\partial t} & =\frac{2 B_{\theta}}{r} \frac{\partial b_{\phi}}{\partial \theta}+\frac{b_{\phi} B_{\theta}}{r} \frac{1}{l_{u}} \\
\frac{1}{l_{u}} & =\frac{d}{d \theta} \ln (r \sin \theta)
\end{aligned}
$$

It is interesting to note that coupled equations of the forms given by Eqs. (4) and (7) can be cast as a 1-D Dirac equation in a Hamiltonian form (see Alicki, 1992, for the case of 

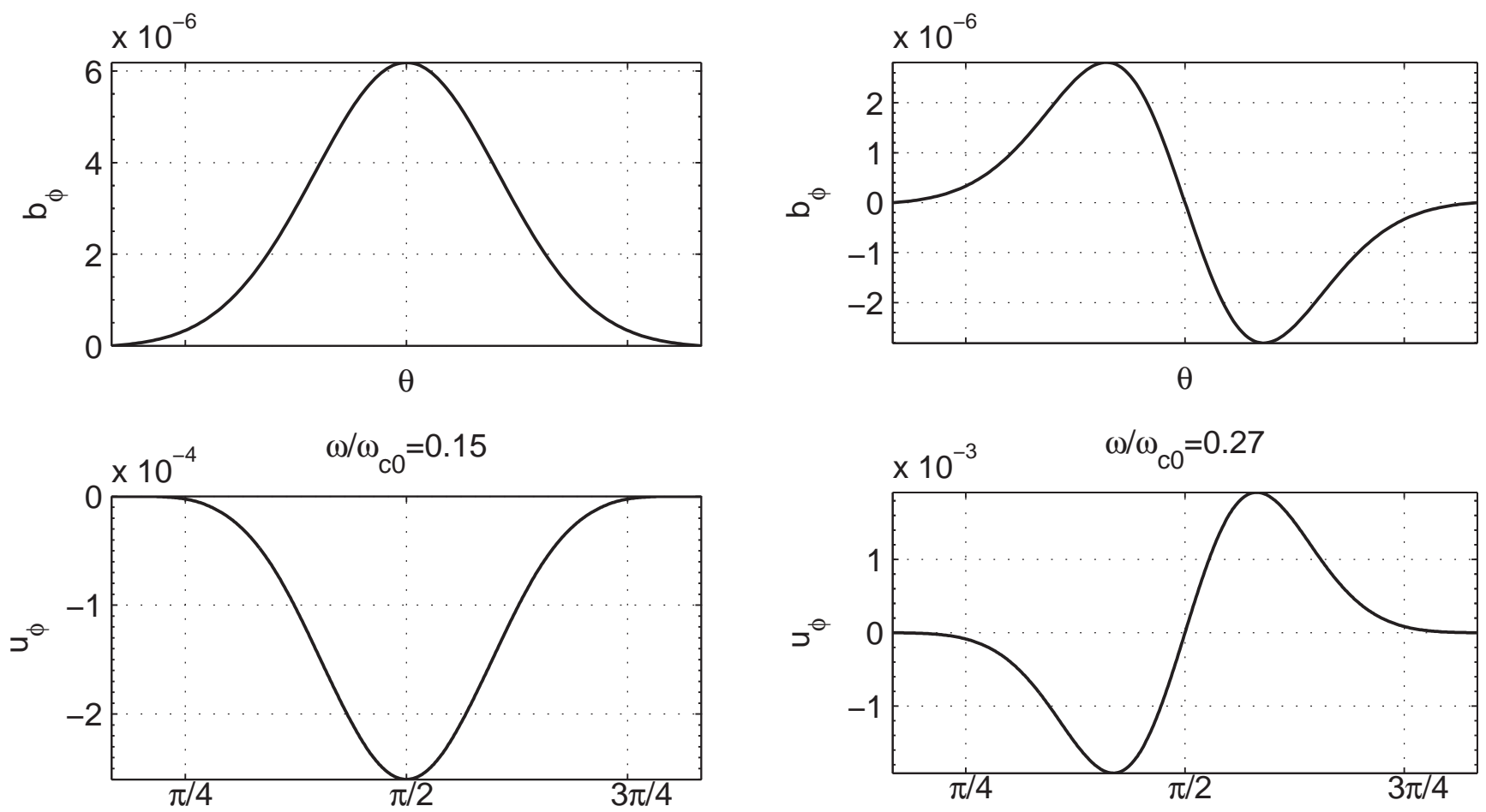

Fig. 3. Solution of the $\mathrm{KG}$ equation for $L=4, p=3$ : the fundamental mode. Both $u_{\phi}$ and $b_{\phi}$ are of arbitrary units.

MHD waves in a one-dimensional solar atmosphere). Here we prefer to develop a Klein-Gordon form for which purpose we note that the two coupled equations for $b_{\phi}$ and $u_{\phi}$ (Eqs. 4 and 7) yield the following wave equations for the perturbations $\left(V^{2}=\left(2 B_{\theta}\right)^{2} / \rho\right)$

$$
\begin{aligned}
\frac{\partial^{2} b_{\phi}}{\partial t^{2}} & =\frac{V^{2}}{r^{2}}\left\{\frac{\partial^{2} b_{\phi}}{\partial \theta^{2}}-\frac{1}{L_{b}} \frac{\partial b_{\phi}}{\partial \theta}+\frac{b_{\phi}}{M_{b}}\right\} \\
\frac{\partial^{2} u_{\phi}}{\partial t^{2}} & =\frac{V^{2}}{r^{2}}\left\{\frac{\partial^{2} u_{\phi}}{\partial \theta^{2}}-\frac{1}{L_{u}} \frac{\partial u_{\phi}}{\partial \theta}+\frac{u_{\phi}}{M_{u}}\right\}
\end{aligned}
$$

in which (all evaluated along $\boldsymbol{B}_{0}$ )

$$
\begin{aligned}
-\frac{1}{L_{b}} & =\frac{d}{d \theta} \ln \left[\frac{B_{\theta}}{\rho r}\left(B_{\theta} g(\theta) r \sin \theta\right)^{1 / 2}\right] \\
\frac{1}{M_{b}} & =\frac{r \rho}{2 B_{\theta}} \frac{d}{d \theta}\left(\frac{B_{\theta}}{\rho r} \frac{1}{l_{u}}\right)+\frac{1}{4} \frac{1}{l_{b}} \frac{1}{l_{u}} \\
-\frac{1}{L_{u}} & =\frac{d}{d \theta} \ln \left[\frac{B_{\theta}}{r}\left(B_{\theta} g(\theta) r \sin \theta\right)^{1 / 2}\right] \\
\frac{1}{M_{u}} & =\frac{r}{2 B_{\theta}} \frac{d}{d \theta}\left(\frac{B_{\theta}}{r} \frac{1}{l_{b}}\right)+\frac{1}{4} \frac{1}{l_{b}} \frac{1}{l_{u}}
\end{aligned}
$$

Fig. 4. Solution of the KG equation for $L=4, p=3$ : the 1 st harmonic.
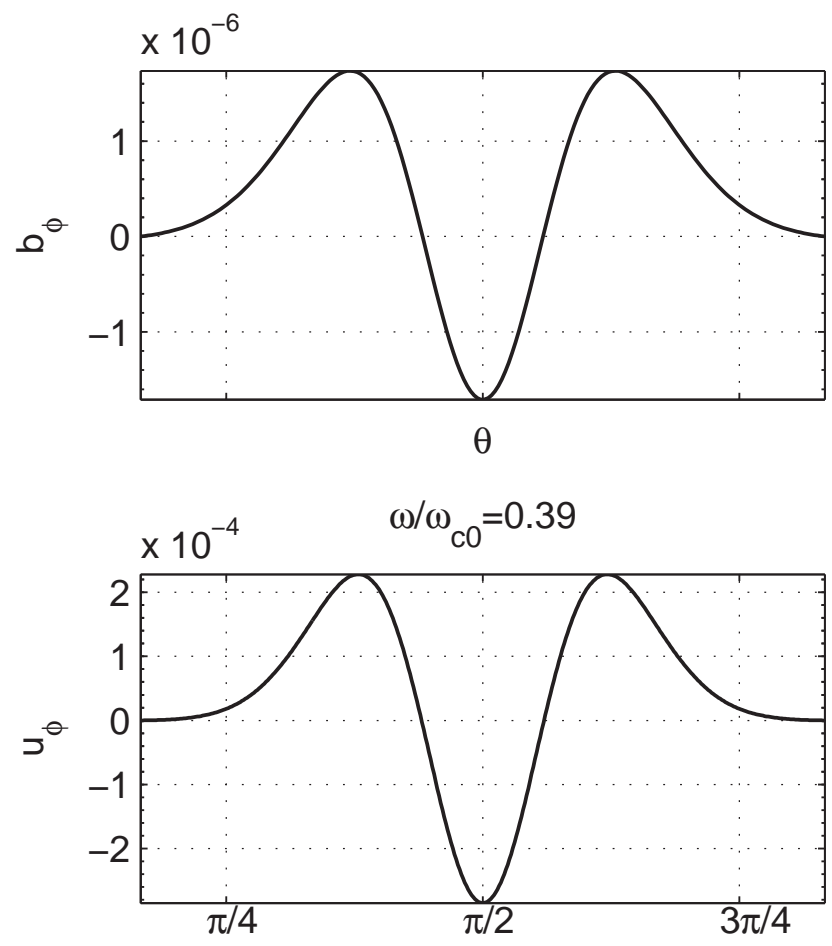

Fig. 5. Solution of the KG equation for $L=4, p=3$ : 2 nd harmonic. 


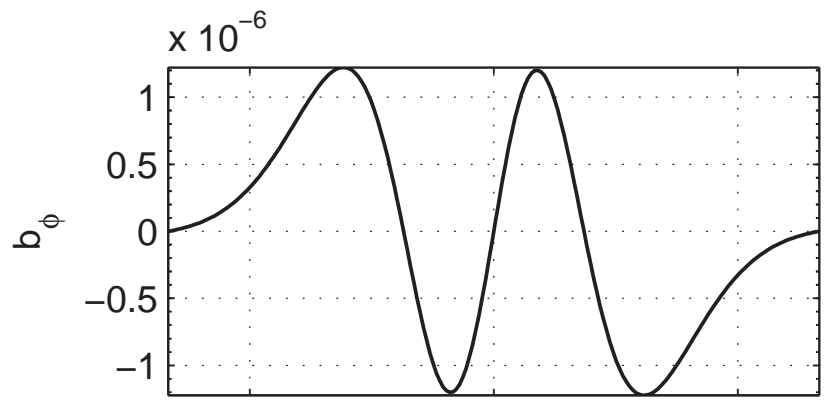

$\theta$

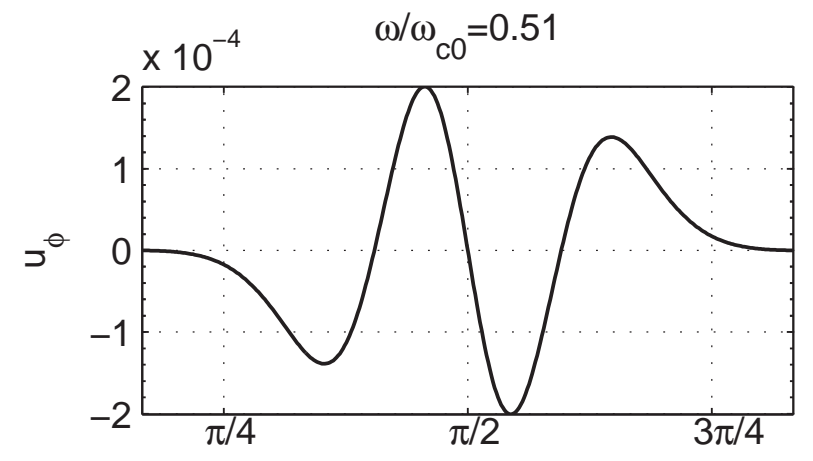

Fig. 6. Solution of the KG equation for $L=4, p=3$ : 3rd harmonic.

\section{The Klein-Gordon form}

\subsection{General case}

Equations of the form (i.e., Eqs. 9 and 10)

$\frac{\partial^{2} \Phi}{\partial t^{2}}=\frac{V^{2}}{r^{2}}\left[\frac{\partial^{2} \Phi}{\partial \theta^{2}}-\frac{1}{\mathcal{L}} \frac{\partial \Phi}{\partial \theta}+\frac{1}{\mathcal{M}} \Phi\right]$

where

$\frac{1}{\mathcal{L}}=-\frac{d}{d \theta} \ln (F(\theta))$

can be transformed into the Klein-Gordon form through the substitution

$\Phi=\phi \exp \int \frac{d \theta}{2 \mathcal{L}}$

to yield

$\frac{\partial^{2} \phi}{\partial t^{2}}+\omega_{c}^{2} \phi=\frac{V^{2}}{r^{2}} \frac{\partial^{2} \phi}{\partial \theta^{2}}$

in which

$\omega_{c}^{2}=\frac{V^{2}}{r^{2}}\left[\frac{1}{2 \mathcal{L}^{2}}\left(1+\mathcal{L}^{\prime}\right)-\frac{1}{\mathcal{M}}\right]$

and the amplitude factor in Eq. (17) becomes

$\exp \int \frac{d \theta}{2 \mathcal{L}}=\frac{1}{F^{1 / 2}(\theta)}$

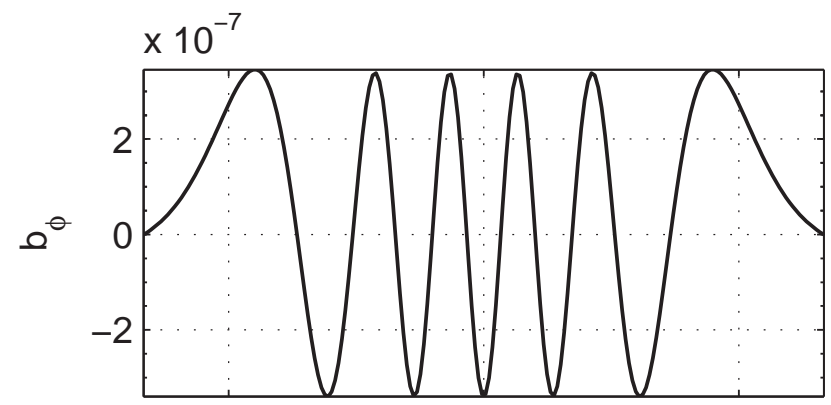

$\theta$

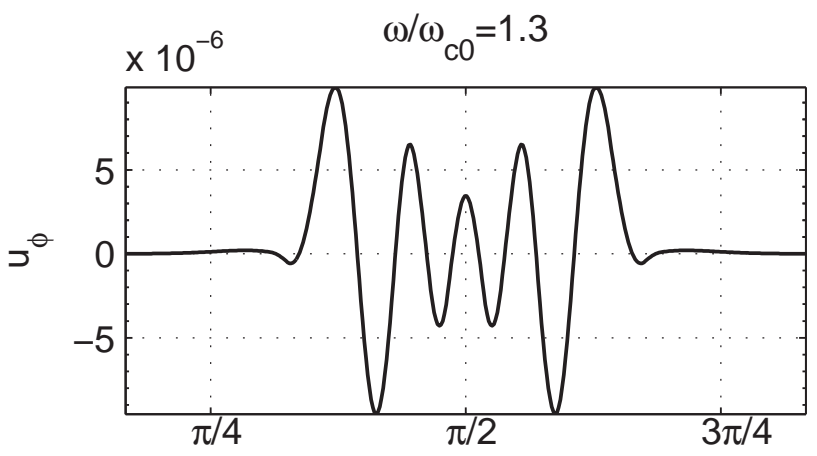

Fig. 7. Solution of the $\mathrm{KG}$ equation for $L=4, p=3$ : higher-order harmonic.

This factor arises from the adiabatic-geometric growth or decay corresponding to conservation of wave energy flux through a flux tube as given by Poynting's theorem (but see below). The quantity $\omega_{c}$, given by Eq. (19), in Eq. (18) is a cut-off frequency as is readily seen by taking a harmonic time variation $\propto \exp (i \omega t)$ for then Eq. (18) becomes

$\frac{d^{2} \phi}{r^{2} d \theta^{2}}=-\frac{\left(\omega^{2}-\omega_{c}^{2}\right)}{V^{2}} \phi \equiv-k^{2} \phi$

An equation of this form possesses propagating-type solutions, provided $\omega>\omega_{c}$ and evanescent solutions for $\omega<\omega_{c}$. If a slowly varying background is assumed, JWKB solutions yield good approximations to the propagating and evanescent behavior. The imposition of boundary conditions (e.g., at the end points of one field line) yield an eigenvalue problem for $k$ (and hence $\omega$ ).

\subsection{Dipole field}

We now evaluate the adiabatic factor $F^{-\frac{1}{2}}(\theta)$ and the cut-off frequency $\omega_{c}$ for the case of a background dipole magnetic field, given by

$\boldsymbol{B}_{0} / B_{0}=2\left(\frac{a}{r}\right)^{3} \cos \theta \hat{r}+\left(\frac{a}{r}\right)^{3} \sin \theta \hat{\theta}$ 
where $a$ is the radius of the Earth, and $B_{0}$ is the equatorial value at the surface of the Earth. The equation of a field line becomes

$\frac{d r}{d \theta}=\frac{r B_{r}}{B_{\theta}}=2 r \cot \theta$

which yields

$r=L a \sin ^{2} \theta$

where $L a$ is the radius at which a given field line cuts the equator (L-shell). Then from Eqs. (5), (6), and (8), we find that

$$
\begin{aligned}
g(\theta) & =1 / \sin ^{4} \theta \\
\frac{1}{l_{b}} & =\frac{d}{d \theta} \ln \left(\frac{1}{\sin ^{9} \theta}\right) \\
\frac{1}{l_{u}} & =\frac{d}{d \theta} \ln \left(\sin ^{3} \theta\right)
\end{aligned}
$$

Substituting these factors into Eqs. (11-14) gives

$$
\begin{aligned}
-\frac{1}{L_{b}} & =\frac{d}{d \theta} \ln \left(\frac{1}{\rho \sin ^{10} \theta}\right)=-10 \cot \theta-\frac{1}{\rho} \frac{d \rho}{d \theta} \\
-\frac{1}{L_{u}} & =\frac{d}{d \theta} \ln \left(\frac{1}{\sin ^{10} \theta}\right)=-10 \cot \theta \\
\frac{1}{M_{u}} & =\frac{9}{2 \sin ^{2} \theta}\left(\frac{11}{2} \cos ^{2} \theta+1\right) \\
\frac{1}{M_{b}} & =-\frac{1}{2 \sin ^{2} \theta}\left(\frac{69}{2} \cos ^{2} \theta+3+3 \cos \theta \sin \theta \frac{d \ln \rho}{d \theta}\right)
\end{aligned}
$$

The adiabatic (growth-decay) amplitude factor (from Eqs. 16 and 11) becomes

$\frac{1}{F^{1 / 2}(\theta)}=\sqrt{\frac{\rho L^{5} a^{1 / 2}}{B_{0}^{3 / 2}}} \sin ^{5} \theta$

The corresponding cut-off frequency from Eq. (19) becomes

$$
\begin{aligned}
\omega_{c}^{2}=\frac{2 B_{0}^{2}}{\rho L^{8} a^{2} \sin ^{14} \theta} & {\left[\left(\frac{10 \cos \theta}{\sin \theta}+\frac{d \ln \rho}{d \theta}\right)^{2}+\frac{26+69 \cos ^{2} \theta}{2 \sin ^{2} \theta}\right.} \\
& \left.+\frac{3 \cos \theta}{\sin \theta} \frac{d \ln \rho}{d \theta}-\frac{d^{2} \ln \rho}{d \theta^{2}}\right]
\end{aligned}
$$

If one assumes that the plasma density $\rho$ varies on a field line according to an inverse radial power law $\propto r^{-p}$ then

$\rho=\rho_{e}(a / r)^{p}=\rho_{e} L^{-p} \sin ^{-2 p} \theta$,

where $\rho_{e}$ is the plasma density at the Earth's ionosphere $(L=$ 1).

Subsequently, we have used Eqs. (28) and (31) for $\mathcal{L}$ and $\mathcal{M}$ to obtain the cut-off frequency in the form

$\omega_{c}^{2}=\frac{\omega_{c 0}^{2}}{2 L^{8-p} \sin ^{(16-2 p)} \theta}\left[\left(269-92 p+8 p^{2}\right) \cos ^{2} \theta+26-4 p\right]$, with $\omega_{c 0}^{2}=2\left(B_{0}^{2} / \rho_{e}\right) / a^{2}$. This equation displays an astonishing variation of $\omega_{c}^{2}$ with $\sin \theta$ along a given field line $(L)$ with $\theta$ going from $\pi / 2$ at the equator to $\sin ^{-1}(\sqrt{1 / L})$ at the Earth's surface (ionosphere).

Relation (35) shows the considerable variation of $\omega_{c}^{2}$ along a field line from its equatorial value to a much higher value in the ionosphere $(L>1)$. This variation of $\omega_{c}^{2}$, as a function of $\theta$ (for various $L$ shell values), along with the adiabatic growth factor, is shown in Fig. 1.

For wave frequencies $\omega$ in excess of (less than) the cut-off frequency the time harmonic Klein-Gordon Eq. (21) yields propagating (evanescent) modes. Thus for a given frequency $\omega$ (eigenvalue) less than its ionosphere value we expect propagating modes equatorward of where $\omega=\omega_{c}$, and evanescent in $\omega<\omega_{c}$ towards the ionosphere. The adiabatic-growth amplitude factor also varies considerably, for example, by a factor of about 4 (30 in Fig. 2) in case of $L=4$.

The corresponding cut-off frequency for the velocity perturbation is

$\omega_{c}^{2}=\frac{B_{0}^{2}}{\rho L^{8} a^{2} \sin ^{16} \theta}\left(101 \cos ^{2} \theta+2\right)$

For a varying density profile given by Eq. (34), the above equation yields

$\omega_{c}^{2}=\frac{\omega_{c 0}^{2}}{2 L^{8-p} \sin ^{(16-2 p)} \theta}\left(101 \cos ^{2} \theta+2\right)$

Note that the cut-off frequencies for the velocity (Eq. 37) and magnetic (Eq. 35) perturbations are not precisely the same since the underlying wave Eqs. (9) and (10) are not identical. It is a common feature of wave propagation in inhomogeneous media that different perturbations satisfy slightly different wave equations. A similar plot with the same format as Fig. 1 for the corresponding toroidal velocity perturbations is shown in Fig. 2. In this case, the corresponding growth-decay factor is

$\frac{1}{F^{1 / 2}(\theta)}=\sqrt{\frac{L^{5} a^{1 / 2}}{B_{0}^{3 / 2}} \sin ^{5} \theta}$

The adiabatic-geometric factors are closely related to the conservation of wave energy in an inhomogeneous plasma embedded in a dipole magnetic field, where the rate of working of $\boldsymbol{J} \cdot \boldsymbol{E}$, namely,

$\mu_{0} \boldsymbol{J} \cdot \boldsymbol{E}=B_{\theta} u_{\phi} \frac{1}{r \sin \theta} \frac{\partial}{\partial \theta}\left(\sin \theta b_{\phi}\right)+B_{r} u_{\phi} \frac{1}{r} \frac{\partial}{\partial r}\left(r b_{\phi}\right)$

is balanced by the divergence of the Poynting flux $(\boldsymbol{E} \times \boldsymbol{B})$, according to (after time averaging)

$\langle\boldsymbol{J} \cdot \boldsymbol{E}\rangle=-\nabla \cdot\langle\boldsymbol{E} \times \boldsymbol{B}\rangle / \mu_{0}$ 


\section{Numerical results and discussion}

The Eqs. (9) and (10) were solved, in turn, in the KG form of Eq. (21), subject to boundary conditions. One physical boundary condition for an ionosphere of infinite conductivity is such that $\boldsymbol{E} \equiv 0$ at both ends of a field line (L-shell). This indicates that $u_{\phi} \equiv 0$ at both ends according to Eq. (1). We solve Eq. (21) for $u_{\phi}$ first, subject to these boundary conditions, with an additional one, $\partial u_{\phi} / \partial \theta=d$, at one end of the field line, necessary for determining the unknown parameter $\omega$. Note that this latter boundary condition will yield a physical unit for $u_{\phi}$ given the value of $d$ from measurements. In the present study, such a value is given arbitrarily, so that the quantities, $u_{\phi}$ and $b_{\phi}$ remain of arbitrary units. Once the $\mathrm{KG}$ equation for $u_{\phi}$ is solved with a determined eigenvalue $\omega$, we solve the KG equation for $b_{\phi}$ with the same $\omega$, subject to boundary conditions $b_{\phi} \equiv 0$ at both ends of a field line. Alternatively, the KG equation for $b_{\phi}$ can be solved first subject to boundary conditions if in practice those are more readily available. We will investigate other solutions subject to different boundary conditions provided by actual observations in future work.

A set of solutions corresponding to different eigenvalues, $\omega$ (normalized by $\omega_{c 0}$ ), and corresponding eigenfunctions, were obtained by selecting different initial guesses of solution. They are shown in Figs. 3-7 for the fundamental, first, second, third harmonic, and a higher-order harmonic, respectively. These eigen-modes with distinct and discrete eigen-frequencies are representative of resonant plasma waves along magetospheric field lines. They seem to correspond to ultra-low-frequency waves, e.g., Pc and Pi pulsations observed in space and on ground, with frequencies ranging from $\mathrm{mHz}$ to a few $\mathrm{Hz}$ (Kivelson and Russell, 1995). In reality, there probably should be a mixture of such wave modes in space and time.

Acknowledgements. One of the authors (JFMcK) acknowledges support from the Pei-Ling Chan Chair of Physics in the University of Alabama in Huntsville.
Topical Editor I. A. Daglis thanks R. Treumann, K.H. Glaßmeier, and another anonymous referee for their help in evaluating this paper.

\section{References}

Alicki, R.: Dirac equations for MHD waves: Hamiltonian spectra and supersymmetry, J. Phys. A: Math. Gen., 25, 6075-6085, 1992.

Allan, W. and Knox, F. B.: A dipole field model for axisymmetric Alfvén waves with finite ionosphere conductivities, Planet. Space Sci., 27, 79-85, 1979.

Chen, L. and Hasegawa, A.: A theory of long period magnetic pulsations-I. Steady state excitation of field line resonance, J. Geophys. Res., 79, 1024-1032, 1974.

Cummings, W. D., O’Sullivan, R. J., and Coleman Jr., P. J.: Standing Alfvén waves in the magnetosphere, J. Geophys. Res., 74, 778-793, 1969.

Kivelson, M. G. and Russell, C. T.: Introduction to space physics, Ch. 11, pp. 330-354, Cambridge University Press, 1995.

McClay, J. F.: On the resonant modes of a cavity and the dynamical properties of micropulsations, Planet. Space Sci., 18, 1673-1690, 1970.

Orr, D. and Matthew, J. A. D.: The variation of geomagetic micropulsation periods with latitude and the plasmapause, Planet. Space Sci., 19, 897-905, 1971.

Radoski, H. R.: A note on oscillating field lines, J. Geophys. Res., 72, 418-419, 1967.

Southwood, D. J.: Some features of field line resonances in the magnetosphere, Planet. Space. Sci., 22, 483, 1974.

Tamao, T.: Transmission and coupling resonance of hydromagnetic disturbances in the non-uniform Earth's magnetosphere, Sci. Rep. Tohoku Univ., Series 5, Geophysics, 17, 43-72, 1965.

Taylor, J. P. H. and Walker, A. D. M.: Accurate approximate formulae for toroidal standing hydromagnetic oscillations in a dipolar geomagnetic field, Planet. Space Sci., 32, 1119-1124, 1984.

Walker, A. D. M.: Modelling of Pc5 pulsation structure in the magnetosphere, Planet. Space Sci., 28, 213-223, 1980. 